\title{
Direction of Arrival Estimation for Uniform Circular Array Based on Monte Carlo Importance Sampling
}

\author{
Peng $\mathrm{Ma}^{1, *}$ and Shiquan Wang ${ }^{2}$ \\ ${ }^{1}$ Department of Combat Support, Bengbu campus of Army Armored Force Institute, Anhui province, P.R.China \\ ${ }^{2}$ Section of Teaching and Research, Bengbu campus of Army Armored Force Institute, Anhui province, P.R.China \\ ${ }^{*}$ Corresponding author
}

\begin{abstract}
A new method of estimating direction of arrival for uniform circular array is presented in this paper. Based on Monte Carlo importance sampling, the proposed method employs a global optimization to maximize the compressed likelihood function at low angular separation to boast relatively improved performance, which guarantees convergence to the global maximum with comparison to other suboptimal algorithm. In addition, the paper testifies the robustness and effectiveness by comparing to other algorithms.
\end{abstract}

Keywords-importance sampling; parameters estimation; uniform circular array

\section{INTRODUCTION}

Many problems in radar, sonar, seismology, and etc., utilize the signals recorded via an array of sensors to perform highresolution estimation of the direction of arrival (DOA) of emitters based on uniform circular array (UCA). For conventional algorithms [1], the model of UCA needs to be transformed to the model of uniform linear array ULA to meet the Vandermonde structure, which induces Coherent noises. Even if de-coherent algorithm could solve the problem concerning coherent noises [2], the accuracy of estimation of direction would be descended. Alternatively, many iterative methods have been proposed, but all require a good initial value [3]. In this paper, for reducing the numbers of parameters of likelihood function, an analytical maximization over the source covariance matrix is employed to optimal the remaining function [4], termed compressed likelihood function, which depends only on the desired arrival angles. In addition, avoiding a multidimensional integration, a non-iterative algorithm implementing Monte Carlo importance sampling for UCA is presented [5], which solve how to get the global optimization estimator without good initial value.

\section{PROBLEM FormULATION}

Considering a UCA composed of $L$ isotropic elements, shown as Figure I. Let $M$ narrow-band signal sources located at angles $\left(\theta_{1}, \varphi_{1}\right)\left(\theta_{2}, \varphi_{2}\right) \ldots\left(\theta_{M}, \varphi_{M}\right)$ respectively impinge on this array, which the radius is $r$ and the center of circle is the reference point. The possible range of the DOA angles $\varphi$ is $[0, \pi / 2]$, and the range of $\theta$ is $[-\pi, \pi]$. The angle between the $i$ th sensor and the axis $\mathrm{X}$ is $\gamma_{i}=2 \pi i / L$, and the vector is $\boldsymbol{p}_{i}=\left(r \cos \gamma_{i}, r \sin \gamma_{i}, 0\right)$. Assuming that signal transfers along $-\boldsymbol{v}$, whose coordinate is $\mathrm{v}=(\sin \varphi \cos \theta, \sin \varphi \sin \theta, \cos \varphi)$. Therefore, the phase delay of the $i$ th sensor is given by $\varphi_{i}=e^{j k p_{i}}=e^{j \xi \cos \left(\theta-\gamma_{i}\right)}$, with $\xi=k_{0} r \sin \varphi$. Accordingly, the steer vector of array is $a(\theta)=a(\xi, \theta)=\left[e^{j \xi \cos \left(\theta-\gamma_{0}\right)}, e^{j \xi \cos \left(\theta-\gamma_{1}\right)}, \mathrm{L}, e^{j \xi \cos \left(\theta-\gamma_{L-1}\right)}\right]$. Here it is assumed that all signal sources are coplanar with array, that is, $\varphi=\pi / 2$. Thus the noisy signal received at the $i$ th sensor at time $t$ is a superposition of the responses to the $M$ individual signals and noise $n_{i}(t)$, and the receiving signal is given by

$$
\boldsymbol{y}_{i}(t)=\sum_{j=1}^{M} a_{i}\left(\theta_{j}\right) \boldsymbol{s}_{j}(t)+\boldsymbol{n}_{i}(t), \quad i=0,1, \mathrm{~L}, L-1
$$

where $\boldsymbol{s}_{i}(t)$ is the $j$ th signal in reaching the array, and $\boldsymbol{n}_{i}(t)$ is the noise sample at each sensor comprising a discrete-time white Gaussian noise process. In essence, the matrix of UCA could be defined as

$$
\boldsymbol{Y}(t)=\boldsymbol{A}(\boldsymbol{\theta}) \boldsymbol{S}(t)+\boldsymbol{N}(t)=\left[\boldsymbol{y}_{0}(t), \boldsymbol{y}_{1}(t), \mathrm{L}, \boldsymbol{y}_{L-1}(t)\right]^{T}
$$

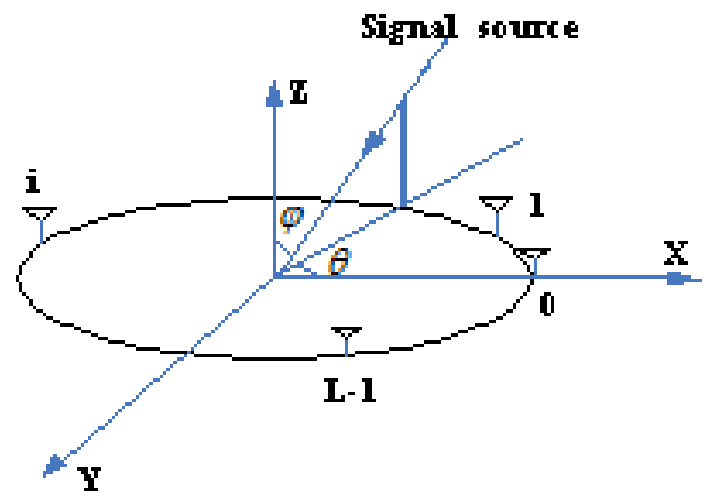

FIGURE I. GEOMETRY OF THE CIRCULAR ARRAY FOR DOA ESTIMATION

where $\boldsymbol{A}(\boldsymbol{\theta})=\left[\boldsymbol{a}\left(\theta_{1}\right), \boldsymbol{a}\left(\theta_{2}\right), \mathrm{L}, \boldsymbol{a}\left(\theta_{M}\right)\right]$ is an $L \times M$ steering matrix whose elements as said above, and $\boldsymbol{S}(t)=\left[\boldsymbol{s}_{1}(t), \boldsymbol{s}_{2}(t), \mathrm{L}, \boldsymbol{s}_{M}(t)\right]^{T}$ is an $M \times 1$ complex signal amplitude vector. The noise is assumed that the sensor noise processes are independent with zero mean and known variance $\sigma^{2}$, where $N(t)=\left[\boldsymbol{n}_{0}(t), \boldsymbol{n}_{1}(t), \mathrm{L}, \boldsymbol{n}_{L-1}(t)\right]^{T}$ is an $L \times 1$ array vector. 


\section{COMPRESSED LIKELIHOOD FUNCTION}

Under the Gaussian random white noise assumption from (2), $\boldsymbol{Y}\left(t_{n}\right)$ for $n=1,2, \mathrm{~L}, N$ is a sum of independent vectors with $\boldsymbol{A}(\boldsymbol{\theta}) \boldsymbol{S}\left(t_{n}\right)$ and $\boldsymbol{N}\left(t_{n}\right)$. As a result, each snapshot has a pdf $\boldsymbol{Y}\left(t_{n}\right) \sim$ $C N\left(\boldsymbol{A}(\boldsymbol{\theta}) \boldsymbol{S}\left(t_{n}\right), \sigma^{2} \boldsymbol{I}_{L}\right)$. The joint pdf of the independent snapshots $\boldsymbol{Y}\left(t_{n}\right)$ for $n=1,2, \mathrm{~L}, N$, is given by

$$
L(f, \boldsymbol{\theta})=-\frac{L N}{2} \ln \left(2 \pi \sigma^{2}\right)-\frac{1}{2 \sigma^{2}} \sum_{n=1}^{N}\left(\boldsymbol{Y}\left(t_{n}\right)-\boldsymbol{A S}\left(t_{n}\right)\right)^{H}\left(\boldsymbol{Y}\left(t_{n}\right)-\boldsymbol{A} \boldsymbol{S}\left(t_{n}\right)\right)
$$

Using the result of [5], the ML estimation of $\hat{\boldsymbol{\theta}}_{\text {mle }}$ can be obtained by maximizing the compressed likelihood function. And the compressed likelihood function $L_{c}(\boldsymbol{\theta})$ can also be written as

$$
L_{c}(\boldsymbol{\theta})=\frac{1}{2 N \sigma^{2}}\left[\sum_{n=1}^{N} \boldsymbol{Y}^{H}\left(t_{n}\right) \boldsymbol{G}(\boldsymbol{\theta}) \boldsymbol{Y}\left(t_{n}\right)\right]+\frac{L N}{2} \ln \left(2 \pi \sigma^{2}\right)
$$

where $\boldsymbol{G}(\boldsymbol{\theta})=\boldsymbol{A}(\boldsymbol{\theta})\left[\boldsymbol{A}^{H}(\boldsymbol{\theta}) \boldsymbol{A}(\boldsymbol{\theta})\right]^{-1} \boldsymbol{A}^{H}(\boldsymbol{\theta})$ is the orthogonal projection matrix.

\section{Global MAXIMIZATION OF LiKELIHOOd FunCTION}

The ML estimation of the DOAs is obtained by maximizing the compressed likelihood function $L_{c}(\theta)$. As said above, a direct implementation requires a multi-dimensional grid search or good initial guess, whose computational complexity increases exponentially with the number of sources or global convergence to the maximum being not guaranteed. To overcome this drawback, it is proposed to use theorem of Pincus [3] that does not require an initial guess of the DOA estimation. Now let $L^{\prime}(\boldsymbol{\theta})=\exp \left(\rho L_{c}(\boldsymbol{\theta})\right)$ and a normalized version of $L^{\prime}(\theta)$ should be

$$
\bar{L}^{\prime}(\boldsymbol{\theta})=\frac{\exp \left[\rho L_{c}(\boldsymbol{\theta})\right]}{\int \mathrm{L} \int \exp \left[\rho L_{c}\left(\boldsymbol{\theta}^{\prime}\right)\right] \mathrm{d} \boldsymbol{\theta}^{\prime}}
$$

Then the function $L^{\prime}(\boldsymbol{\theta})$ has all the properties of a pdf, in that it is nonnegative and integrates to one. The term $L^{\prime}(\boldsymbol{\theta})$ is considered as a pseudo-pdf in $\boldsymbol{\theta}$. With this definition, Pincus's theorem may be interpreted from (5) as a method for computing the global maximum of $L_{c}(\theta)$ as the mean of the $L^{\prime}(\boldsymbol{\theta})$ as $\rho \rightarrow \infty$. Then estimate of $\boldsymbol{\theta}$ can be found from

$$
\hat{\boldsymbol{\theta}}=\frac{1}{K} \sum_{k=1}^{K} \boldsymbol{\theta}_{k}
$$

where $\boldsymbol{\theta}_{k}$ is the $k$ th realization of the "random" vector $\boldsymbol{\theta}$ distributed according to $\bar{L}^{\prime}(\boldsymbol{\theta})$ and $\rho$ is sufficiently large. Computing $\hat{\boldsymbol{\theta}}$ by (12) requires generation of $\boldsymbol{\theta} \sim \bar{L}^{\prime}(\boldsymbol{\theta})$.

\section{IMPORTANCE SAMPLING}

Importance sampling has been shown to be a powerful tool for computing a multidimensional integral [4]. The expectation can be found to any desired degree of accuracy (for large enough $K$ ) by the Monte Carlo sample mean estimate

$$
\hat{E}_{p}\left[h(\boldsymbol{\theta}) \frac{\eta(\boldsymbol{\theta})}{p(\boldsymbol{\theta})}\right]=\frac{1}{K} \sum_{k=1}^{K} h\left(\boldsymbol{\theta}_{k}\right) \frac{\eta\left(\boldsymbol{\theta}_{k}\right)}{p\left(\boldsymbol{\theta}_{k}\right)}
$$

where $\eta(\boldsymbol{\theta})$ and $p(\boldsymbol{\theta})$ are assumed to be pseudo-pdfs. The function $p(\theta)$ is usually called the importance function, which can at times be chosen to be some simple function of $\boldsymbol{\theta}$, so that realizations of $\theta$ can be easily generated.

\section{CHOICE OF IMPORTANCE FUNCTION}

Since the goal is to choose an importance function similar to the normalized version of $\exp \left[\rho L_{c}(\theta)\right]$, but that is separable, only the first term of the compressed likelihood function can be considered as given in (4). It can be separated by letting $\boldsymbol{A}^{H}(\boldsymbol{\theta}) \boldsymbol{A}(\boldsymbol{\theta})$ be replaced by $L \boldsymbol{I}_{M}$. Note that for large signal-tonoise ratio and well separated $\theta_{m}$, this is a good approximation. The first term of the compressed likelihood function then becomes

$$
\begin{aligned}
& \frac{1}{2 N \sigma^{2}} \sum_{n=1}^{N} \boldsymbol{Y}^{H}\left(t_{n}\right) \boldsymbol{G}(\boldsymbol{\theta}) \boldsymbol{Y}\left(t_{n}\right) \\
& \quad=\frac{1}{2 N \sigma^{2}} \sum_{n=1}^{N} \boldsymbol{Y}^{H}\left(t_{n}\right) \boldsymbol{A}(\boldsymbol{\theta})\left[\boldsymbol{A}^{H}(\boldsymbol{\theta}) \boldsymbol{A}(\boldsymbol{\theta})\right]^{-1} \boldsymbol{A}^{H}(\boldsymbol{\theta}) \boldsymbol{Y}\left(t_{n}\right) \\
& \quad \approx \frac{1}{2 N \sigma^{2}} \sum_{n=1}^{N} \boldsymbol{Y}^{H}\left(t_{n}\right) \boldsymbol{A}(\boldsymbol{\theta})\left(\frac{\boldsymbol{I}_{M}}{L}\right) \boldsymbol{A}^{H}(\boldsymbol{\theta}) \boldsymbol{Y}\left(t_{n}\right)
\end{aligned}
$$

by letting $\left[\boldsymbol{A}^{H}(\boldsymbol{\theta}) \boldsymbol{A}(\boldsymbol{\theta})\right]^{-1} \approx\left(\boldsymbol{I}_{M} / L\right)$. Therefore the importance function can be chosen as

$$
\exp \left(\frac{\rho}{2 N \sigma^{2}} \sum_{n=1}^{N} \boldsymbol{Y}^{H}\left(t_{n}\right) \boldsymbol{A}(\boldsymbol{\theta})\left(\frac{\boldsymbol{I}_{M}}{L}\right) \boldsymbol{A}^{H}(\boldsymbol{\theta}) \boldsymbol{Y}\left(t_{n}\right)\right)
$$

Since from expression of $\boldsymbol{A}(\boldsymbol{\theta})$, that (9) becomes

$$
\begin{aligned}
\exp \left[\frac{\rho}{2 N \sigma^{2} L} \sum_{n=1}^{N} \sum_{m=1}^{M}\left|\sum_{i=0}^{L-1} y_{i}\left(t_{n}\right) \exp \left(j \xi \cos \left(\theta_{m}-\gamma_{i}\right)\right)\right|^{2}\right] \\
=\prod_{m=1}^{M} \exp \left[\frac{\rho}{2 N \sigma^{2} L} \sum_{n=1}^{N} \mid \sum_{i=0}^{L-1} y_{i}\left(t_{n}\right) \exp \left(\left.j \xi \cos \left(\theta_{m}-\gamma_{i}\right)\right|^{2}\right]\right. \\
=\prod_{m=1}^{M} \exp \left(\rho I_{Y}\left(\theta_{m}\right)\right)
\end{aligned}
$$

where $I_{Y}\left(\theta_{m}\right)=\frac{1}{2 N \sigma^{2}} \sum_{n=1}^{N}\left[\frac{1}{L}\left|\sum_{i=0}^{L-1} y_{i}\left(t_{n}\right) \exp \left(j \xi \cos \left(\theta_{m}-\gamma_{i}\right)\right)\right|^{2}\right]$.

Hence, the importance function is chosen to be 


$$
p(\theta)=\frac{\exp \left(\rho_{1} I_{Y}(\theta)\right)}{\int \exp \left(\rho_{1} I_{Y}\left(\theta^{\prime}\right)\right) d \theta^{\prime}}
$$

\section{ESTIMATION OF DIRECTIONS}

The global optimization theorem requires evaluation of the means of $\theta_{1}, \theta_{2}, \mathrm{~L}, \theta_{M}$, distributed according to a pseudo-pdf. And to avoid estimation bias, the linear mean will be replaced by the circular mean to estimate the DOA. The circular mean for a random variable $\theta$ defined on the interval $[-\pi, \pi]$ is

$$
E(\boldsymbol{\theta})=\angle \int_{-\pi}^{\pi} \exp (j \boldsymbol{\theta}) p(\boldsymbol{\theta}) d \boldsymbol{\theta}
$$

where $\angle$ denotes the angle in radians and $p(\boldsymbol{\theta})$ is the pdf. Key idea in defining a circular mean is to average position vectors. Hence the circular sample mean of the data is defined as

$$
\boldsymbol{\theta}=\angle \frac{1}{K} \sum_{k=1}^{K} \exp (j \boldsymbol{\theta})
$$

The use of (13) alleviates the estimator bias. This is valid as a consequence of the invariance properties of the maximum likelihood estimator [5].Thus, $\hat{\theta}_{m, m l e}$ is found by letting

$$
\begin{aligned}
h(\boldsymbol{\theta}) & =\exp \left(j \theta_{m}\right) \\
\zeta(\boldsymbol{\theta}) & =\frac{\exp \left[\rho L_{c}(\boldsymbol{\theta})\right]}{\int \exp \left[\rho L_{c}\left(\boldsymbol{\theta}^{\prime}\right)\right] d \boldsymbol{\theta}^{\prime}} \\
p(\boldsymbol{\theta}) & =\prod_{m=1}^{M} \frac{\exp \left[\rho_{1} I_{Y}\left(\theta_{m}\right)\right]}{\int \exp \left[\rho_{1} I_{Y}\left(\theta_{m}^{\prime}\right)\right] d \theta_{m}^{\prime}}
\end{aligned}
$$

and using the definition of circular mean estimate to yield

$$
\hat{\theta}_{m, m l e}=\angle \frac{1}{K} \sum_{k=1}^{K} \frac{\zeta\left(\boldsymbol{\theta}_{k}\right)}{p\left(\boldsymbol{\theta}_{k}\right)} \exp \left(j\left[\boldsymbol{\theta}_{k}\right]_{m}\right)
$$

for $m=1,2, \mathrm{~L}, M$. Note that since only the angle of the complex quantity in (15) will be gotten, the equivalent estimator is

$$
\hat{\theta}_{m, m l e}=\angle \frac{1}{K} \sum_{k=1}^{K} w\left(\boldsymbol{\theta}_{k}\right) \exp \left(j\left[\boldsymbol{\theta}_{k}\right]_{m}\right)
$$$$
w(\boldsymbol{\theta})=\frac{\exp \left[\rho L_{c}(\boldsymbol{\theta})\right]}{\prod_{m=1}^{M} \exp \left[\rho_{1} I_{Y}\left(\theta_{m}\right)\right]}
$$
where ${ }_{m=1} \exp \left[\rho_{1} I_{Y}\left(\theta_{m}\right)\right]$. To overcome the difficulty of
producing large values at times, ${ }^{w(\theta)}$ can be replaced by ${ }^{\prime}(\boldsymbol{\theta})$
}

$$
\begin{aligned}
w^{\prime}\left(\boldsymbol{\theta}_{k}\right)= & \exp \left[\rho L_{c}\left(\boldsymbol{\theta}_{k}\right)-\rho_{1} \sum_{m=1}^{M} I_{Y}\left(\left[\theta_{k}\right]_{m}\right)\right. \\
& \left.-\max \left(\rho L_{c}\left(\boldsymbol{\theta}_{k}\right)-\rho_{1} \sum_{m=1}^{M} I_{Y}\left(\left[\theta_{k}\right]_{m}\right)\right)\right]
\end{aligned}
$$

and $\boldsymbol{\theta}_{k}$ is the $k$ th realization of $\boldsymbol{\theta}$.Thus $w(\boldsymbol{\theta})$ in (16) can be replaced by $w^{\prime}(\boldsymbol{\theta})$ so that

$$
\hat{\theta}_{m, m l e}=\angle \frac{1}{K} \sum_{k=1}^{K} w^{\prime}\left(\boldsymbol{\theta}_{k}\right) \exp \left(j\left[\boldsymbol{\theta}_{k}\right]_{m}\right) \quad m=1,2, \mathrm{~L}, M
$$

\section{SIMULATION ANALYSIS}

In this section, two DOA estimation algorithms: MODE by Stoica and Sharman [6], and EMML by Miller and Fuhrman [7] are compared with the proposed method above, because two algorithms are representative of the different methods being studied in detail for DOA estimation based on UCA. For all the simulations to follow, an $L=9$ element UCA has been chosen. Also, the number of realizations necessary for the ISML is always fixed at $K=2000$.

Two sources at $\theta_{1}=-5^{\circ} \quad \theta_{2}=10^{\circ}$ are chosen, $N=100$ snapshots and $S N R=0 d B$. The Root Mean Square Error (RMSE) versus the values of these parameters is shown in Figure II and III. From Figure II, it is seen that there is little dependence on $\rho$, as long as it is at least equal to ten, and we see that there is some dependence on $\rho_{1}$.If $\rho_{1}$ is too large, and then the importance function may become too narrow. As a result, not all possible angles will be generated, resulting in a biased estimator. As expected, the importance function must be appropriately chosen. This choice appears to be sufficiently robust in that, as will be shown, the performance is nearly optimal even as the SNRs, and actual angles vary over a wide range.

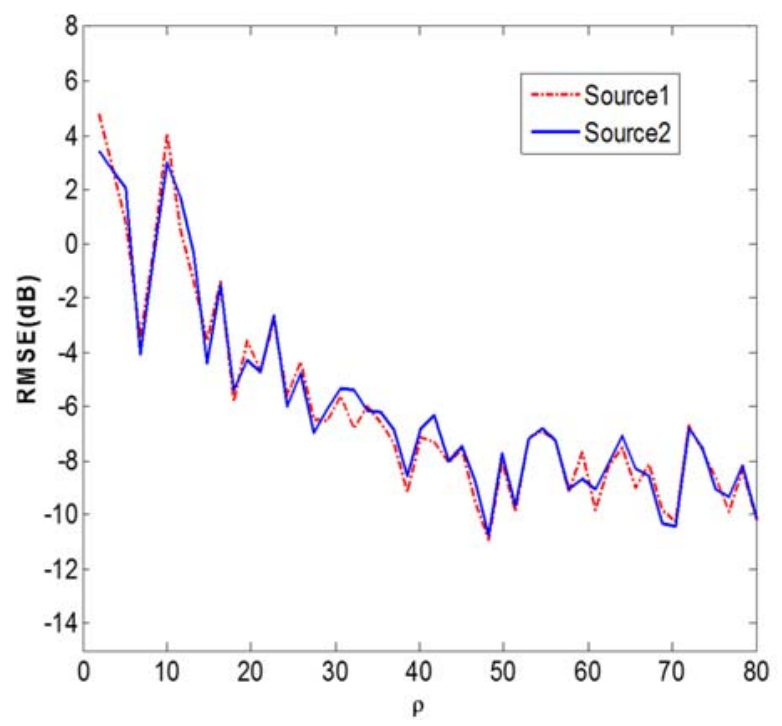

FIGURE II. SENSITIVITY OF RMSE TO PARAMETER ${ }^{\rho}$ (WITH $^{\rho_{1}=1}$ ) 


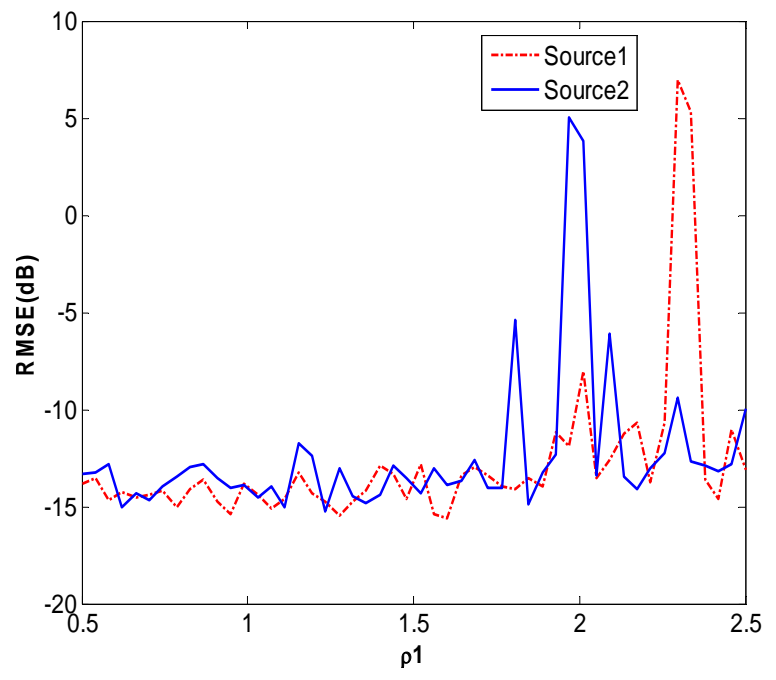

FIGURE III. SENSITIVITY OF RMSE TO PARAMETER $\rho_{1}$ ( WITH $\rho=80$ )

In order to give the greatest advantage to the iterative methods, the initial estimates is made to be close to the true angles by choosing the initial angle estimates as $\hat{\theta}_{1}=-2^{\circ}$ and $\hat{\theta}_{2}=7^{\circ}$. The results are shown in Figure IV. It is seen that there is little difference between EMML and ISML. MODE has worse performance for its process of de-coherent. This is as expected since all the iterative algorithms are able to attain the maximum of the likelihood function if they are provided with an initial estimate close to it. However, even for good initial angle estimates, if the sources are spaced within a beam-width at $\theta_{1}=-5^{\circ}$ and $\theta_{2}=-3^{\circ}$ with good initial angle estimates of $\hat{\theta}_{1}=-7^{\circ}$ and $\hat{\theta}_{2}=-1^{\circ}$, the results are considerably poorer, as shown in Figure V. The ISML (with $\rho=300$ and $\rho_{1}=1$ ) has better performance than the other approaches.

To study what happens when there is no good initials, the simulations are studied for two closely spaced sources at $\theta_{1}=-5^{\circ}$ and $\theta_{2}=2^{\circ}$ with the number of snapshots $N=100$ and $S N R=0 d B$. In the simulation, the initial estimate for $\theta_{2}=2^{\circ}$ was fixed at $\hat{\theta}_{2}=5^{\circ}$, close to the true value, and the other initial estimate for $\theta_{1}=-5^{\circ}$ varied. The results are shown in Figure VI. It is seen that EMML is very sensitive to the initial estimate, while MODE is less so. Hence, if the initial estimate is far from the true value, very poor performance can be expected. Only the ISML can guarantee robustness to the initial estimates, as there is no dependence.
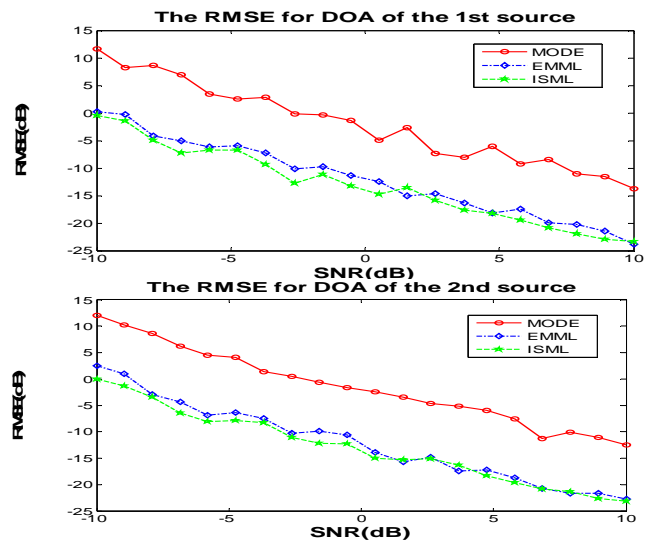

FIGURE IV. RMSE COMPARISON VERSUS SNR FOR WIDELY SPACED SOURCES AND GOOD INITIAL ANGLE ESTIMATES.
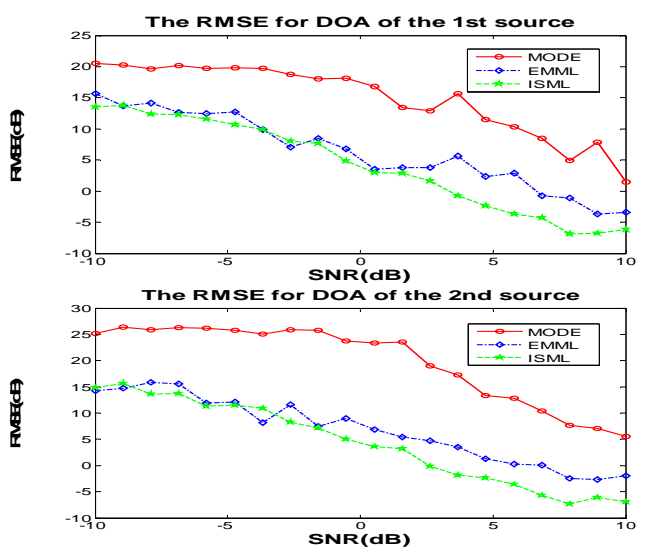

FIGURE V. RMSE COMPARISON VERSUS SNR FOR CLOSELY SPACED SOURCES AND GOOD INITIAL ANGLE ESTIMATES.
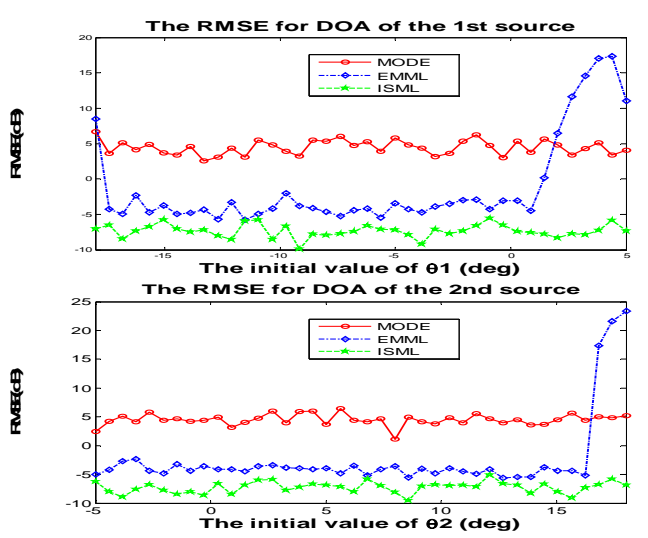

FIGURE VI. RMSE COMPARISON VERSUS SNR FOR WIDELY SPACED SOURCES AND GOOD INITIAL ANGLE ESTIMATES.

According to the simulation conditions given above, the computational complexity is next assessed. This was done by determining the computer running time for an average of 100 estimates. The results are given in Table I. It is seen that the most computational efficient approaches is MODE. Both EMML and ISML are significantly more computationally intensive. In a practical implementing method of the ISML, it may be necessary to speed up the computation by a more efficient implementing method. 
TABLE I. AVERAGE COMPUTATIONAL TIME IN SECONDS

\begin{tabular}{|c|c|c|}
\hline Method & TIME & $\begin{array}{c}\text { RELATIVE } \\
\text { SPEED }\end{array}$ \\
\hline MODE & 0.316 & 1 \\
\hline EMML & 13.641 & 43.16 \\
\hline ISML & 15.084 & 47.73 \\
\hline
\end{tabular}

\section{CONCLUSION}

A computationally modest technique (with respect to a multidimensional grid search) to implement the MLE to estimate DOA of UCA has been developed. It is important to note that technique does not require any initial guess of the angle estimates but still performs better than the iterative ML methods. It should also be mentioned that the importance sampling approach, since it operates on a compressed likelihood function, does not require any special assumptions about the array to be satisfied. It can be applied to nonuniformly spaced and/or multidimensional arrays. In summary, all three methods perform well in an environment with a high SNR and large number of snapshots. However, only the ISML algorithm produces accurate estimates for closely spaced sources at low SNR and for a small number of snapshots. This advantage will only increase as the number of sources increases.

\section{REFERENCES}

[1] T. Basikolo, K. Ichige, and H. Arai, "A Novel Mutual Coupling Compensation Method for Underdetermined Direction of Arrival Estimation in Nested Sparse Circular Arrays," IEEE Transactions on Antennas and Propagation, 2018, vol. 66, pp. 909-921.

[2] J Guojun; M Xingpeng, and L Yongtan, "ADirection-of-arrival estimation for uniform circular arrays under small sample size," Journal of Systems Engineering and Electronics, vol.27, 2016, pp: 1142 - 1150 .

[3] J H Lee and S H Jo. "On initialization of ML DOA cost function for UCA,” Progress In Electromagnetics Research M, 2018, Vol 3, No. 5, pp: 91-102.

[4] S. Kanakaraj, S. M. Nair, and Saidalavi Kalady, "SAR Image Super Resolution using Importance Sampling Unscented Kalman Filter," IEEE Journal of Selected Topics in Applied Earth Observations and Remote Sensing, 2018, vol.11, pp: $562-571$.

[5] W. Huigang, S. Kay, and S. Saha, "An Importance Sampling Maximum Likelihood Direction of Arrival Estimator," IEEE Transactions on Signal Processing, 2008, vol.56, pp: $5082-5092$.

[6] R Y Rubinstein. "Simulation and the Monte Carlo Method," New York: Wiley, 1981, CH. 3.

[7] S. Kay. "Comments on frequency estimation by linear prediction," IEEE Trans. Acoust., Speech, Signal Process, Apr, 1979, Vol. ASSP-27, PP: 198-199. 